\title{
Escala de actitud religiosa en estudiantes de Medicina Humana
}

\author{
María Teresa Rivera-Encinas*1, 2, a, b, Jeff Huarcaya-Victoria 1, 3, a
}

RESUMEN

Objetivo: Establecer la validez y fiabilidad de la Escala de actitud religiosa (EAR) en una muestra de estudiantes de medicina de una universidad privada del Perú.

Materiales y métodos: Estudio observacional y transversal. Se aplicó la EAR a una muestra de 687 estudiantes de medicina para valorar las propiedades psicométricas.

Resultados: El puntaje medio de la EAR fue de 62,97 $\pm 15,74$. Destaca la diferencia en variables género ( $p=0,011)$, año de estudio $(p=0,035)$, y religión $(p=0,000)$. Se encontraron 3 factores con autovalores de $9,34,1,53$ y 1,02 respectivamente. Cada uno aporta el 35,22 \%, 17,68 \% y 17,09\% de la varianza total explicada en la rotación, que es, en conjunto, el $70 \%$ de la varianza total. La EAR tiene una consistencia interna aceptable (alfa de Cronbach=0,94), pero en su versión de 14 ítems aumenta a 0,951.

Conclusiones: Se puede inferir que la EAR, en su versión de 14 ítems, cuenta con propiedades psicométricas adecuadas para poder evaluar la actitud de la población de estudiantes peruanos de Medicina Humana hacia la religión.

Palabras clave: Religión y Medicina; Espiritualidad; Educación médica; Estudiantes de Medicina; Perú (Fuente: DeCS BIREME).

\section{Religious attitude scale in students of human medicine}

\section{ABSTRACT}

Objective: To establish the validity and reliability of the Religious Attitude Scale (RAS) in a sample of medical students from a Peruvian private university.

Materials and methods: An observational and cross-sectional study was conducted. The RAS was administered to a sample of 687 medical students to assess the psychometric properties.

Results: The mean score of the RAS was $62.97 \pm 15.74$. Differences in gender $(p=0.011)$, academic year $(p=0.035)$ and religion $(p=0.000)$ stand out. Three factors with eigenvalues of 9.34, 1.53 and 1.02 were found, each contributing $35.22 \%, 17.68 \%$ and $17.09 \%$ of the total variance explained in the rotation, which accounts for $70 \%$ of the total variance. The RAS has an acceptable internal consistency (Cronbach's alpha $=0.94$ ); however, in its 14 -item version it increases to 0.951 .

Conclusions: It can be concluded that the RAS, in its 14-item version, has adequate psychometric properties to evaluate the attitude of the population of Peruvian students of human medicine toward religion.

Keywords: Religion and medicine; Spirituality; Education, medical; Students, medical; Peru (Source: MeSH NLM).

1. Universidad de San Martín de Porres, Facultad de Medicina, Centro de Investigación en Salud Pública. Lima, Perú.

2. Instituto Nacional de Salud Mental "Honorio Delgado - Hideyo Noguchi". Lima, Perú.

3. Hospital Nacional Guillermo Almenara Irigoyen, Departamento de Psiquiatría, Servicio de Psiquiatría General. Lima, Perú.

a. Médico cirujano, Psiquiatra.

b. Magíster en Salud Pública con mención en Salud Ocupacional.

*Autor corresponsal. 


\section{INTRODUCCIÓN}

La religión y la espiritualidad han estado conectadas a la medicina durante siglos. Los hospitales y universidades originalmente estuvieron asociados a tendencias religiosas por templos y sociedades (1). A pesar de ello, a inicios de siglo XX, hubo una separación entre religión y medicina, justificada por la concentración en los descubrimientos científicos. Sin embargo, desde la década de 1960, varios estudios epidemiológicos comenzaron a mostrar el impacto de la espiritualidad y religiosidad en la salud del paciente, lo que le permite sobrellevar mejor los eventos negativos de la vida y tener actitudes más positivas en esos momentos ${ }^{(2)}$. Asimismo, a lo largo del tiempo, se han establecido las necesidades espirituales de los pacientes en el entorno de atención médica pero, actualmente, los médicos aún no las satisfacen, lo que demuestra que se presta poca atención a las creencias y actitudes religiosas de los pacientes en la práctica general diaria ${ }^{(3)}$.

Muchos profesionales del sector salud consideran que hablar de religión y espiritualidad es beneficioso e, incluso, responsable de resultados clínicos positivos, lo que motiva la creación de la expresión "Evidencia basada en la espiritualidad", que trata de explicar, mediante investigaciones científicas, la asociación posiblemente causal entre espiritualidad y buena salud (4). Por tal motivo, muchas escuelas de medicina alrededor del mundo, como en Estados Unidos y Canadá, comenzaron a incorporar cursos sobre espiritualidad y salud en sus planes curriculares, asociados a las pautas éticas que se vieron necesarias de implementar en la atención integral y centrada en el paciente ${ }^{(5,6)}$.

Es necesario reconocer que la espiritualidad y la religiosidad son conceptos polisémicos y, en diferentes estudios, es difícil definirlos operacionalmente, a pesar de que tienen constructos que pueden sobreponerse. Por ello, y para efectos de este proyecto, definimos a la espiritualidad como la vivencia interna de trascendencia y disposición para interactuar con el resto a nivel emocional y conductual; y a la religiosidad como la identificación de la persona con una institución religiosa, donde el sujeto se conduce de acuerdo a creencias y prácticas normadas por la organización ${ }^{(7)}$.

Lamentablemente, en las facultades de Medicina Humana de Latinoamérica y, específicamente, del Perú, estos temas no se investigan ni se toman con la importancia debida, lo que se ve reflejado en los pocos estudios y las pocas escalas validadas que evalúan la religiosidad o espiritualidad en personal de salud y estudiantes de medicina, a pesar de que los últimos son reconocidos como aprendices del buen trato hacia el paciente.

Debido a que no contamos con escalas validadas en el
Perú que valoren la actitud religiosa, decidimos realizar este estudio que tiene como principal objetivo establecer la validez y fiabilidad de la escala de actitud religiosa (EAR) en una muestra de estudiantes de medicina de una universidad privada del Perú.

\section{MATERIALES Y MÉTODOS}

Diseño y población del estudio

Estudio observacional y transversal, que buscó explorar las propiedades psicométricas de la escala de actitud religiosa (EAR) en una muestra de estudiantes de medicina, además de correlacionar los niveles de religiosidad con algunas variables personales. En el estudio de la elaboración de la EAR realizado en población mexicana se reportó una consistencia interna de $0,94^{(8)}$, por lo que se esperó encontrar en nuestro estudio una consistencia interna igual o superior. Además, se valoró la hipótesis de encontrar mayores niveles de religiosidad en mujeres, casados, afiliados a una religión, el deseo de seguir una especialidad centrada en el paciente y el deseo de estudiar medicina.

En el estudio participaron 687 alumnos de medicina de primer a sexto año de la Facultad de Medicina Humana de la Universidad de San Martín de Porres (FMH-USMP) durante el período académico 2017-II. La recolección de datos se realizó durante el mes de noviembre en las diferentes aulas en las que se dictaron las clases teóricas, la muestra fue por conveniencia. No se tomó en cuenta a los alumnos de séptimo año (internado médico) por temas logísticos.

\section{Variables y mediciones}

La actitud religiosa se valoró con la Escala de actitud religiosa (EAR), diseñada en México por Orozco-Parra y Domínguez-Espinosa ${ }^{(8)}$, que tomaron como base los trabajos realizados por Francis en las poblaciones cristiana, judía, hindú e islámica (9-12). Inicialmente, esta escala contaba con 19 ítems, luego de los estudios de validez y fiabilidad se redujo a 17, de los cuales 6 se deben puntuar en sentido invertido. Con esta reducción a 17 ítems se logró una consistencia interna aceptable (alfa de Cronbach=0,94). En el análisis factorial se encontró que los 17 ítems podrían ser explicados en 2 factores con autovalores de 9,5 y 1,33 , que aportaron $36,33 \%$ y $27,37 \%$ de la varianza, respectivamente. Sin embargo, los autores de la escala recomiendan que una solución a un único factor sería suficiente, el cual explicaría el 55,9 \% de la varianza total.

En un estudio previo encontramos que la consistencia interna de la EAR en una muestra de 142 médicos de un hospital general fue alta (alfa de Cronbach $=0,92)^{(13)}$. Los autores de la EAR recomiendan que debería utilizarse, de preferencia, en personas que practiquen alguna religión teísta, ya que la validación de esta escala se limita a la población católica ${ }^{(8)}$. 
Junto a la EAR, se entregó una ficha de recolección de datos sociodemográficos, en la cual figuraban las siguientes variables: edad, género, año de estudio, trabajo, estado civil, filiación religiosa, especialidad que desea seguir ("vinculada al paciente" o "vinculada a la tecnología"), deseo de estudiar medicina, y si contó con un modelo profesional en el trato con el paciente.

\section{Análisis estadístico}

Se realizaron técnicas de estadística descriptiva para todas las variables. Se reportó la media y desviación estándar $(\mathrm{X} \pm \sigma)$, mínimo y máximo para las variables numéricas y análisis de frecuencias para las variables categóricas.

Se realizó un análisis exploratorio de las variables cuantitativas para determinar su normalidad. La relación entre la EAR con las variables cualitativas se evaluó mediante la prueba $U$ de Mann-Whitney, debido a que no se cumplieron los supuestos de normalidad.

Se llevó a cabo un análisis de la validez de la EAR, para lo cual, primero se evaluó la viabilidad de realizar un análisis factorial mediante el criterio de adecuación factorial de Keiser-Meyer-Olkin (KMO) y la prueba de esfericidad de Barlett. El número de factores se determinó con el criterio de autovalor $>1$, el método de análisis de componentes principales, y luego se ajustó mediante rotación Varimax con normalización de Kaiser.

La fiabilidad se evaluó a través de un análisis de consistencia interna con alfa de Cronbach. Se eliminaron los ítems necesarios para mejorar la consistencia interna de la EAR, y luego se realizó, nuevamente, un análisis de validez.

El nivel alfa se determinó antes del análisis en 0,05. Los análisis se realizaron con ayuda del programa estadístico SPSS de IBM versión 23.

\section{Consideraciones éticas}

Se respetó la confidencialidad de los participantes en cumplimiento de la Declaración de Helsinki. Se informó a cada uno de la voluntariedad de participar en el estudio. Además, se contó con la autorización del Comité de Ética de la FMH-USMP.

\section{RESULTADOS}

Entre los 687 alumnos de medicina encuestados, la edad promedio fue de 19,91 $\pm 2,82$ años (la mínima fue de 15 y la máxima de 41). Las demás características sociodemográficas las podemos encontrar en la tabla 1.

Tabla 1. Características sociodemográficas

\begin{tabular}{|cc}
\multicolumn{1}{c}{ Variable } & $\mathbf{n}(\%)$ \\
\hline Edad, años, media $( \pm \sigma)$ [rango] & $19,91( \pm 2,82)[15-41]$ \\
Género & $274(39,9)$ \\
Masculino & $413(60,1)$ \\
Femenino & \\
Año de estudio & $228(33,2)$ \\
Primero & $205(29,8)$ \\
Segundo & $78(11,4)$ \\
Tercero & $31(4,5)$ \\
Cuarto & $69(10)$ \\
Quinto & $76(11,1)$ \\
Sexto & \\
Trabajo & $48(7)$ \\
Sí & $639(93)$ \\
No & $672(97,8)$ \\
Estado civil & $11(1,6)$ \\
Soltero & $4(0,6)$ \\
Casado & \\
Otro & \\
\hline
\end{tabular}




\begin{tabular}{|lc|}
\multicolumn{1}{|c}{ Variable } & $\mathbf{n}(\%)$ \\
\hline Religión & \\
Católico & $519(75,5)$ \\
Protestante / Evangélico & $44(6,4)$ \\
Agnóstico & $57(8,3)$ \\
Ateo & $36(5,2)$ \\
Otro & $31(4,5)$ \\
Especialidad que desea seguir & \\
Centrada en el paciente & $433(63)$ \\
Centrada en la tecnología & $216(31,4)$ \\
No sabe & $38(5,5)$ \\
¿Deseó estudiar medicina? & $649(94,5)$ \\
Sí & $38(5,5)$ \\
No & \\
Modelo en el trato al paciente & $497(72,3)$ \\
Sí & $190(27,7)$ \\
No &
\end{tabular}

б: Desviación estándar

El puntaje medio de la EAR en los 687 alumnos fue de $62,97 \pm$ 15,74. El análisis comparativo, según las variables estudiadas para la puntuación total de la EAR, se encuentra en la tabla
2. Las únicas diferencias estadísticamente significativas se observan en el puntaje total de la EAR en las variables género $(p=0,011)$, año de estudio $(p=0,035)$, y religión $(p=0,000)$.

Tabla 2. Análisis comparativo según las variables estudiadas para las puntuaciones totales de la EAR en 687 estudiantes de Medicina Humana

\begin{tabular}{|c|c|c|c|c|}
\hline \multirow{2}{*}{ Variable } & \multirow{2}{*}{ n (\%) } & \multicolumn{2}{|c|}{ EAR } & \multirow{2}{*}{$p$} \\
\hline & & $X \pm \sigma$ & $\operatorname{Me}(I Q R)$ & \\
\hline Género & & & & $0,011^{*}$ \\
\hline Masculino & $274(39,9)$ & $61 \pm 16,45$ & $62(21)$ & \\
\hline Femenino & $413(60,1)$ & $64,28 \pm 15,12$ & $67(23)$ & \\
\hline Año de estudio & & & & $0,035^{* *}$ \\
\hline Primero & $228(33,2)$ & $65,02 \pm 13,61$ & $67(20)$ & \\
\hline Segundo & $205(29,8)$ & $61,51 \pm 17,81$ & $64(26)$ & \\
\hline Tercero & $78(11,4)$ & $60,77 \pm 15,94$ & $60,5(22)$ & \\
\hline Cuarto & $31(4,5)$ & $59,48 \pm 13,25$ & $62(18)$ & \\
\hline Quinto & $69(10)$ & $61,26 \pm 15,85$ & $63(20)$ & \\
\hline Sexto & $76(11,1)$ & $66 \pm 15,57$ & $69(21)$ & \\
\hline Trabajo & & & & $0,914^{*}$ \\
\hline Sí & $48(7)$ & $62,81 \pm 17,07$ & $67(23)$ & \\
\hline No & $639(93)$ & $62,98 \pm 15,65$ & $66(23)$ & \\
\hline Estado civil & & & & $0,369^{*}$ \\
\hline Soltero & $672(97,8)$ & $62,88 \pm 15,82$ & $66(23)$ & \\
\hline Casado & $11(1,6)$ & $67,91 \pm 11,97$ & $68(24)$ & \\
\hline
\end{tabular}




\begin{tabular}{|c|c|c|c|c|}
\hline \multirow{2}{*}{ Variable } & \multirow{2}{*}{ n (\%) } & \multicolumn{2}{|c|}{ EAR } & \multirow{2}{*}{$p$} \\
\hline & & $X \pm \sigma$ & $\operatorname{Me}(I Q R)$ & \\
\hline Religión & & & & $0,000^{* *}$ \\
\hline Católico & $519(75,5)$ & $64,84 \pm 13,90$ & $67(20)$ & \\
\hline Protestante / Evangélico & $44(6,4)$ & $67,59 \pm 14,66$ & $69(26)$ & \\
\hline Agnóstico & $57(8,3)$ & $54,30 \pm 19,50$ & $57(32)$ & \\
\hline Ateo & $36(5,2)$ & $44,03 \pm 17,23$ & $41(24)$ & \\
\hline Otro & $31(4,5)$ & $63,13 \pm 17,17$ & $63(26)$ & \\
\hline Especialidad que desea seguir & & & & $0,107^{* *}$ \\
\hline Centrada en el paciente & $433(63)$ & $62,9 \pm 16,33$ & $66(23)$ & \\
\hline Centrada en la tecnología & $216(31,4)$ & $64,02 \pm 14,34$ & $65,5(22)$ & \\
\hline No sabe & $38(5,5)$ & $57,79 \pm 15,74$ & $60(22)$ & \\
\hline ¿Deseó estudiar medicina? & & & & $0,879^{*}$ \\
\hline Sí & $649(94,5)$ & $63,02 \pm 15,68$ & $66(23)$ & \\
\hline No & $38(5,5)$ & $62,08 \pm 16,80$ & $68(22)$ & \\
\hline Modelo en el trato al paciente & & & & $0,293^{*}$ \\
\hline Sí & $497(72,3)$ & $63,40 \pm 15,41$ & $67(23)$ & \\
\hline No & $190(27,7)$ & $61,85 \pm 16,56$ & $64(22)$ & \\
\hline
\end{tabular}

X: media, $\sigma$ : desviación estándar, Me: mediana, IQR: rango intercuartílico

* U de Mann-Whitney

${ }^{* *}$ Prueba de Kruskal Wallis

Validez de la EAR

Para comprobar la pertinencia de realizar un análisis de la validez de la EAR se calculó la medida de adecuación muestral KMO y se confirmó dicho resultado con la prueba de esfericidad de Barlett, las cuales fueron satisfactorias (prueba de esfericidad de Bartlett: $x^{2}=8709,554, p=$ 0,000 ; medida $\mathrm{KMO}=0,955$ ).

En el análisis factorial de los 17 ítems de la EAR, a través de una extracción de componentes principales con rotación Varimax y normalización de Kaiser, se encontraron 3 factores con autovalores de 9,34, 1,53 y 1,02 respectivamente, cada uno aportó el 35,22 \%, 17,68 \% y 17,09 \% de la varianza total explicada en la rotación, y fueron, en conjunto, el 70 $\%$ de la varianza total.

\section{Fiabilidad de la EAR}

Encontramos que la EAR tiene una consistencia interna aceptable (alfa de Cronbach=0,94). Este valor aumenta si se eliminan los ítems 7, 12 y 14 (Tabla 3). Los demás ítems tuvieron un índice de homogeneidad >0,2, por lo que no fue necesario eliminar ninguno más. Al eliminar los ítems indicados encontramos una mejor consistencia interna (alfa de Cronbach=0,951).

Tabla 3. Estadísticos de correlación y de eliminación de ítem del alfa de Cronbach

\begin{tabular}{|lccc} 
& $\begin{array}{c}\text { Correlación } \\
\text { total de ítems } \\
\text { corregida }\end{array}$ & $\begin{array}{c}\text { Correlación } \\
\text { múltiple al } \\
\text { cuadrado }\end{array}$ & $\begin{array}{c}\text { Alfa de Cronbach } \\
\text { si el ítem se ha } \\
\text { suprimido }\end{array}$ \\
\hline 1. Dios me ayuda a llevar una vida mejor. &, 795 &, 762 &, 940 \\
\hline 2. Me gusta mucho aprender sobre Dios. &, 753 &, 670 &, 941 \\
3. Dios significa nada para mí (R). &, 573 &, 455 &, 944 \\
4. Creo que Dios ayuda a la gente. &, 762 &, 657 &, 940
\end{tabular}




\begin{tabular}{|c|c|c|c|}
\hline & $\begin{array}{l}\text { Correlación } \\
\text { total de ítems } \\
\text { corregida }\end{array}$ & $\begin{array}{l}\text { Correlación } \\
\text { múltiple al } \\
\text { cuadrado }\end{array}$ & $\begin{array}{l}\text { Alfa de Cronbach } \\
\text { si el ítem se ha } \\
\text { suprimido }\end{array}$ \\
\hline 5. La oración me ayuda mucho. & ,817 & ,777 & ,939 \\
\hline 6. Pienso que orar es una buena cosa. & ,790 & ,729 & 940 \\
\hline $\begin{array}{l}\text { 7. Pienso que los textos religiosos (por ejemplo: la } \\
\text { Biblia, el Corán, el Torá) son anticuados (R). }\end{array}$ & ,403 & ,296 & ,948 \\
\hline 8. Dios es muy real para mí. & ,826 & ,745 & ,939 \\
\hline 9. Pienso que orar sirve para nada (R). & ,710 &, 581 & ,942 \\
\hline 10. La religión me ayuda a llevar una mejor vida. & ,819 & ,717 & ,939 \\
\hline 11. Encuentro difícil creer en Dios (R). & 691 &, 579 & ,942 \\
\hline $\begin{array}{l}\text { 12. Pienso que los rituales religiosos son una pérdida } \\
\text { de tiempo (R). }\end{array}$ &, 539 & ,452 & ,945 \\
\hline $\begin{array}{l}\text { 13. Para mí es importante practicar mi religión/ } \\
\text { creencias espirituales. }\end{array}$ & ,701 & ,619 & ,942 \\
\hline 14. La religión es relevante en el mundo moderno. & ,435 & ,292 & ,947 \\
\hline $\begin{array}{l}\text { 15. Pienso que ir al templo (por ejemplo: iglesia, } \\
\text { sinagoga, mezquita) es una pérdida de tiempo (R). }\end{array}$ & ,606 &, 524 & ,944 \\
\hline $\begin{array}{l}\text { 16. Mis creencias religiosas realmente dan forma a mi } \\
\text { enfoque total de la vida. }\end{array}$ &, 775 & ,718 & ,940 \\
\hline 17. La espiritualidad es importante en mi vida. & ,755 & ,680 & ,941 \\
\hline
\end{tabular}

(R): ítem con puntaje invertido. Los ítems eliminados se encuentran en negrita.

\section{Análisis factorial exploratorio de la EAR reducida}

Al eliminar los ítems 7, 12 y 14 obtuvimos una prueba de esfericidad de Bartlett: $x 2=7863,408, p=0,000$ y medida $\mathrm{KMO}=0,953$. Al análisis factorial de los 14 ítems de la EAR, a través de una extracción de componentes principales con rotación Varimax y normalización de Kaiser, se encontraron 2 factores con autovalores de 8,673 y 1,15 respectivamente, cada uno aporta el $44,92 \%$ y $25,24 \%$ de la varianza total explicada en la rotación y, en conjunto, son el 70,1\% de la varianza total (Tabla 4).

Tabla 4. Análisis factorial de 14 ítems de la EAR luego de la rotación

\begin{tabular}{ll} 
& Factores \\
& $\mathbf{1}$ \\
\hline 16. Mis creencias religiosas realmente dan forma a mi enfoque total de la vida. &, 824 \\
2. Me gusta mucho aprender sobre Dios &, 818 \\
17. La espiritualidad es importante en mi vida. &, 799 \\
10. La religión me ayuda a llevar una mejor vida. &, 781 \\
13. Para mí es importante practicar mi religión/creencias espirituales. &, 775 \\
5. La oración me ayuda mucho. &, 774 \\
8. Dios es muy real para mí. &, 732 \\
1. Dios me ayuda a llevar una vida mejor. &, 730 \\
4. Creo que Dios ayuda a la gente. &, 724 \\
6. Pienso que orar es una buena cosa. &, 722
\end{tabular}




\begin{tabular}{lr} 
& Factores \\
& $\mathbf{1}$ \\
\hline 3. Dios significa nada para mí (R). &, 799 \\
9. Pienso que orar sirve para nada (R). &, 738 \\
11. Encuentro difícil creer en Dios (R). &, 737 \\
15. Pienso que ir al templo (por ejemplo: iglesia, sinagoga, mezquita) es una &, 726 \\
\hline
\end{tabular}

Método de extracción: análisis de componentes principales. Método de rotación: Varimax con normalización Kaiser. No se consideraron valores absolutos < 0,50.

Factor 1: "actitudes positivas hacia la religión", Factor 2: "actitudes negativas hacia la religión".

Con la EAR reducida a 14 ítems, encontramos un puntaje medio en los 687 alumnos de $52,54 \pm 13,65$. El análisis comparativo según las variables estudiadas para la puntuación total de la EAR reducida la encontramos en la tabla 5. Las únicas diferencias estadísticamente significativas ocurren en el puntaje total de la EAR en las variables género $(p=0,000)$, religión $(p=0,000)$, y modelo en el trato al paciente $(p=0,034)$.

Tabla 5. Análisis comparativo según las variables estudiadas para las puntuaciones totales de la EAR en 687 estudiantes de Medicina Humana

\begin{tabular}{|c|c|c|c|c|}
\hline \multirow{2}{*}{ Variable } & \multirow{2}{*}{ n (\%) } & \multicolumn{2}{|c|}{ EAR-14 ítems } & \multirow{2}{*}{$p$} \\
\hline & & $X \pm \sigma$ & $\operatorname{Me}(I Q R)$ & \\
\hline Género & & & & $0,000^{*}$ \\
\hline Masculino & $274(39,9)$ & $48,20 \pm 14,46$ & $50,5(20)$ & \\
\hline Femenino & $413(60,1)$ & $55,42 \pm 12,29$ & $58(19)$ & \\
\hline Año de estudio & & & & $0,108^{* *}$ \\
\hline Primero & $228(33,2)$ & $53,99 \pm 11,91$ & $56(18)$ & \\
\hline Segundo & $205(29,8)$ & $52,02 \pm 15,12$ & $54(22)$ & \\
\hline Tercero & $78(11,4)$ & $49,79 \pm 14,45$ & $50,5(19)$ & \\
\hline Cuarto & $31(4,5)$ & $50,03 \pm 12,19$ & $52(17)$ & \\
\hline Quinto & $69(10)$ & $51,22 \pm 13,75$ & $54(20)$ & \\
\hline Sexto & $76(11,1)$ & $54,64 \pm 13,61$ & $58,5(18)$ & \\
\hline Trabajo & & & & $0,629^{*}$ \\
\hline Sí & $48(7)$ & $51,40 \pm 14,33$ & $53(20)$ & \\
\hline No & $639(93)$ & $52,63 \pm 13,61$ & $55(20)$ & \\
\hline Estado civil & & & & $0,764^{*}$ \\
\hline Soltero & $672(97,8)$ & $52,51 \pm 13,66$ & $55(20)$ & \\
\hline Casado & $11(1,6)$ & $52,91 \pm 15,63$ & $57(27)$ & \\
\hline Religión & & & & $0,000^{* *}$ \\
\hline Católico & $519(75,5)$ & $55,23 \pm 10,71$ & $56(16)$ & \\
\hline Protestante / Evangélico & $44(6,4)$ & $61,41 \pm 9,12$ & $63(14)$ & \\
\hline Agnóstico & $57(8,3)$ & $36,82 \pm 13,37$ & $37(21)$ & \\
\hline Ateo & $36(5,2)$ & $29,31 \pm 13,74$ & $28(16)$ & \\
\hline Otro & $31(4,5)$ & $50,84 \pm 15,73$ & $48(27)$ & \\
\hline
\end{tabular}




\begin{tabular}{|c|c|c|c|c|}
\hline \multirow{2}{*}{ Variable } & \multirow{2}{*}{ n (\%) } & \multicolumn{2}{|c|}{ EAR-14 ítems } & \multirow{2}{*}{$p$} \\
\hline & & $X \pm \sigma$ & $M e(I Q R)$ & \\
\hline Especialidad que desea seguir & & & & $0,070^{* *}$ \\
\hline Centrada en el paciente & $433(63)$ & $53,27 \pm 13,80$ & $56(20)$ & \\
\hline Centrada en la tecnología & $216(31,4)$ & $51,39 \pm 13,25$ & $53(18)$ & \\
\hline No sabe & $38(5,5)$ & $50,74 \pm 13,95$ & $54,5(19)$ & \\
\hline ¿Deseó estudiar medicina? & & & & $0,823^{*}$ \\
\hline Sí & $649(94,5)$ & $52,63 \pm 13,49$ & $55(20)$ & \\
\hline No & $38(5,5)$ & $50,97 \pm 16,33$ & $55,5(24)$ & \\
\hline Modelo en el trato al paciente & & & & $0,034^{*}$ \\
\hline Sí & $497(72,3)$ & $53,22 \pm 13,45$ & $56(20)$ & \\
\hline No & $190(27,7)$ & $50,77 \pm 14,04$ & $53(17)$ & \\
\hline
\end{tabular}

$X:$ media, $\sigma:$ desviación estándar, Me: mediana, IQR: rango intercuartílico

* U de Mann-Whitney

** Prueba de Kruskal Wallis

\section{DISCUSIÓN}

De los resultados obtenidos en esta investigación, podemos inferir que la EAR, en su versión de 14 ítems, cuenta con propiedades psicométricas adecuadas para poder evaluar la actitud de la población de estudiantes peruanos de medicina humana hacia la religión. Encontramos una fiabilidad superior a la reportada en el estudio de creación y validación de dicha escala en México, y también a la generalmente obtenida en otras escalas que evalúan la religiosidad ${ }^{(8)}$.

A diferencia de los anteriores estudios, encontramos que la EAR no se puede reducir a un solo factor, explicándose la varianza en dos factores: actitud positiva hacia la religión y actitud negativa hacia la religión.

Nuestra investigación concuerda con la literatura respecto a que la religiosidad es mayor en mujeres que ene hombres. Esto puede deberse a algunos rasgos de personalidad y a que en las mujeres existen mayores niveles de optimismo, satisfacción con la vida, salud física y mental $(8,14,15)$.

No resulta sorprendente que el gran porcentaje de alumnos que se identificaba con una religión, en este caso la católica, tenga un grado alto de religiosidad. En estudios se muestra que existe una asociación entre la pertenencia a algún grupo religioso y variables positivas, como salud física, salud mental o felicidad, lo que a su vez, podría influir en el manejo de los pacientes ${ }^{(14)}$. En otro trabajo, se reitera que la religión y la espiritualidad pueden llevar a la promoción de la salud mental de los estudiantes y protegerlos contra anomalías de la sociedad. En otras palabras, pertenecer a un grupo religioso puede hacer que una persona tenga emociones positivas como protección contra enfermedades mentales y también físicas ${ }^{(15)}$.

Encontramos que los estudiantes que refirieron tener un modelo a seguir en el trato a los pacientes puntuaron más alto en la EAR, por lo que podemos inferir que la imagen de alguien que genera admiración puede ser determinante para el trato hacia el paciente. Recordemos que el aprendizaje observacional es tan importante como el verbal y que puede ser una de las principales causas del continuo buen o mal trato hacia los pacientes ${ }^{(16)}$.

Otro punto que centra nuestra atención es que observamos niveles elevados de religiosidad en los primeros tres años de carrera médica, bloque que pertenece a ciencias básicas, y luego comienza a disminuir. Esto podría implicar que hay una menor humanización en el manejo hacia el paciente, por la premisa de que a mayor religiosidad se da un trato más empático hacia él ${ }^{(16)}$. Además, coincide con el inicio de aprendizaje quirúrgico, por lo que podría también establecerse modelos de los docentes menos empáticos.

Lamentablemente, estos resultados reflejan un problema mayor que afecta a muchas facultades de medicina de Latinoamérica, con respecto a la enseñanza de espiritualidad, religiosidad y salud en los estudiantes de medicina. Se ve que existe una gran brecha en la enseñanza de estos temas entre alumnos de dicha carrera británicos y norteamericanos, con respecto a los latinoamericanos (17). En un estudio realizado en Brasil se evidencia este hecho por tener pocos cursos dedicados a espiritualidad y salud, 
a pesar que la mayoría de autoridades encuestadas, como decanos de las escuelas de medicina afirman que estos temas son importantes en la enseñanza durante la carrera de medicina ${ }^{(18)}$.

Desde nuestro punto de vista, el cambio en la malla curricular es urgente. Se debe enfocar en la integración de temas que se refieran a empatía, espiritualidad y salud, ya que existe evidencia que los pacientes desean más atención a los aspectos religiosos y espirituales en la salud y enfermedad, pero parece que no hay mayor involucramiento de los médicos en la atención primaria de la salud ${ }^{(19,20)}$. Más aún, porque son los mismos estudiantes quienes reconocen la importancia de conocer estos temas. En el estudio multicéntrico SBRAME (Spirituality and Brazilian Medical Education) en el que participaron 12 escuelas de medicina brasileñas, la mayoría de los estudiantes de medicina creían que la espiritualidad tiene un impacto en la salud de los pacientes $(71,2 \%)$ y que este impacto fue positivo $(68,2 \%)$. La mayoría también quería abordar temas de espiritualidad y religiosidad en su práctica clínica $(58,0 \%$ ) y lo consideró relevante $(75,3 \%)$, aunque casi la mitad $(48,7 \%)$ no estaba preparada para hacerlo. Con respecto a su capacitación, la mayoría de los $E M$ informaron que nunca habían participado en una actividad de "espiritualidad y salud" $(81,0 \%)$ y que sus instructores médicos nunca o rara vez habían abordado este problema $(78,3 \%)$. La mayoría también creía que debían estar preparados para abordar los problemas espirituales relacionados con la salud de sus pacientes $(61,6 \%)$ y que este contenido debería incluirse en el currículo médico $(62,6 \%)^{(21)}$. Estos cambios curriculares deben realizarse tomando en cuenta la etnicidad y la cultura de los estudiantes de medicina, ya que estos pueden tener una influencia importante sobre cómo se aborda la espiritualidad y religiosidad en la práctica médica ${ }^{(22) .}$

Este estudio debe ser entendido dentro de sus potenciales limitaciones metodológicas. La EAR, al ser una escala autoadministrada, podría generar un sesgo de deseabilidad. Además, nuestra muestra estuvo constituida principalmente por alumnos católicos, por lo que estos no podrían ser extrapolados a otro tipo de población.

Vemos que existe una gran brecha entre las expectativas de los alumnos y su real formación en espiritualidad y religiosidad. Existe gran trabajo por hacer para consolidar y enseñar la parte humanística de la carrera de medicina humana. Por tal motivo, recomendamos la evaluación de los planes curriculares de las escuelas de Medicina Humana y considerar la inserción de estos tópicos para el manejo de los futuros profesionales hacia los usuarios de la atención.

Finalmente, en estudios posteriores es necesario realizar un análisis factorial confirmatorio. Asimismo, se requieren más investigaciones longitudinales que evalúen el impacto de la inclusión de estos temas, no solo en la formación del alumno, sino como indicadores de calidad en los distintos servicios de salud.

Contribuciones de los autores: MRE y JHV han participado en la concepción del artículo, recolección de datos y aprobación de la versión final.

\section{REFERENCIAS BIBLIOGRÁFICAS}

1. Koenig $\mathrm{H}$. Religion and medicine I: Historical background and reasons for separation. Int J Psychiatry Med. 2000; 30(4): 38598.

2. Kuyvenhoven MM, De Wit NJ, Kuyck WG. Do doctors pay attention to the religious beliefs of their patients? A survey amongst Dutch GPs. Family Practice. 2000; 17(3): 230-2.

3. Best $M$, Butow $P$, Olver I. Doctors discussing religion and spirituality: a systematic literature review. Palliative Medicine. 2016; 30(4): 327-37.

4. Saad M, Masiero D, Battistella LR. Espiritualidade baseada em evidências. Acta Fisiátrica. 2001; 8(3): 107-12.

5. Lucchetti G, Lucchetti AL, Puchalski CM. Spirituality in medical education: global reality?. J Relig Health. 2012; 51(1): 3-19.

6. Puchalski CM, Larson DB. Developing curricula in spirituality and medicine. Acad Med. 1998; 73(9): 970-4.

7. Miller WR, Thoresen CE. Spirituality, religion, and health. An emerging research field. Am Psychol. 2003; 58(1): 2435.

8. Orozco-Parra C, Domínguez-Espinoza C. Diseño y validación de la escala de actitud religiosa. Revista de Psicología. 2014; 23(1): 3-11.

9. Francis LJ, Enger T. The Norwegian translation of the Francis Scale of Attitude toward Christianity. Scand J Psychol. 2002; 43(5): 363-7.

10. Francis LJ, Katz YJ. Measuring attitude toward judaism: the internal consistency reliability of the Katz-Francis Scale of Attitude toward Judaism. Ment Health Religion Cult. 2007; 10(4): 309-24.

11. Francis LJ, Santosh YR, Robbins M, Vij S. Assessing attitude toward hinduism: the Santosh-Francis Scale. Ment Health Relig Cult. 2008; 11(6): 609-21.

12. Francis LJ, Sahin A, Al-Failakawi F. Psychometric properties of two islamic measures among young adults in Kuwait: the Sahin-Francis Scale of Attitude toward Islam and the Sahin Index of Islamic Moral Values. J Muslim Ment Health. 2008; 3(1): 9-24.

13. Huarcaya-Victoria J, Sancho Dávila-Palacios J, De la Cruz-Oré J. Relación entre la actitud hacia la homosexualidad y actitud religiosa en médicos de un hospital general. An Fac med. 2018; 79(2): 138-43.

14. Naceur F. Religiosity and its association with positive and negative emotions among college students from Algeria $\mathrm{AU}$ Abdel-Khalek, Ahmed M. Ment Health Relig Cult. 2007; 10(2): 159-70.

15. Pour F, Rasekhi S. Correlation between religious beliefs with mental health and Academic performance in medical 
students. Int J Med Sci Public Health. 2016; 5(1): 1-6.

16. Huarcaya-Victoria J, Rivera-Encinas MT. Evaluación de la empatía en alumnos de medicina humana de una universidad privada del Perú. Educ Med. 2018.

17. Harbinson MT, Bell D. How should teaching on whole person medicine, including spiritual issues, be delivered in the undergraduate medical curriculum in the United Kingdom?. BMC Med Educ. 2015; 15: 96.

18. Lucchetti G, Lucchetti AL, Espinha DC, de Oliveira LR, Leite JR, Koenig HG. Spirituality and health in the curricula of medical schools in Brazil. BMC Med Educ. 2012; 12: 78.

19. Monroe MH, Bynum D, Susi B, Phifer N, Schultz L, Franco M, et al. Primary care physician preferences regarding spiritual behavior in medical practice. Arch Intern Med. 2003; 163(22): 2751-6.

20. Anandarajah G, Roseman JL. A qualitative study of physicians' views on compassionate patient care and spirituality: medicine as a spiritual practice?. R I Med J (2013). 2014; 97(3): 17-22.

21. Lucchetti G, De Oliveira LR, Koenig HG, Leite JR, Lucchetti AL. Medical students, spirituality and religiosity-results from the multicenter study SBRAME. BMC Med Educ. 2013; 13: 162.

22. Lucchetti G, Ramakrishnan P, Karimah A, Oliveira GR, Dias A, Rane A, et al. Spirituality, religiosity, and health: a comparison of Physicians' Attitudes in Brazil, India, and Indonesia. Int J Behav Med. 2016; 23(1): 63-70.
Fuentes de financiamiento:

Este artículo ha sido financiado por los autores.

\section{Conflictos de interés:}

Los autores declaran no tener ningún conflicto de interés.

\section{Correspondencia:}

María Teresa Rivera Encinas

Dirección: Av. Alameda del Corregidor, 1531, La Molina. Lima.

Teléfono: (511) 3653640

Correo electrónico: mriverae@usmp.pe

Recibido: 01 de setiembre de 2019 .

Evaluado: 04 de setiembre de 2019

Aprobado: 14 de octubre de 2019.

(c) La revista. Publicado por Universidad de San Martín de Porres, Perú. (c) $)_{\text {Br }}$ Licencia de Creative Commons Artículo en acceso abierto bajo términos de Licencia Creative Commons Atribución 4.0 Internacional. (http://creativecommons.org/licenses/by/4.0/)

\section{ORCID iDs}

María Teresa Rivera-Encinas Jeff Huarcaya-Victoria https://orcid.org/0000-0002-1415-9210

https://orcid.org/0000-0003-4525-9545 